\title{
¿CÓMO REPORTAN LOS NIÑOS LAS VOCES PROPIAS Y AJENAS EN SUS RELATOS DE EXPERIENCIA PERSONAL? UN ENFOQUE DEL DESARROLLO DE LA COMPETENCIA COMUNICATIVA-NARRATIVA INFANTIL ${ }^{1}$
}

\author{
Yamileth María Betancourt Córdoba \\ Universidad del Atlántico (Colombia) \\ yamilethbetancourt@mail.uniatlantico.edu.co
}

\author{
Melisa Caro Cervantes \\ Corporación Universitaria Rafael Núñez (Colombia) / Universidad del Atlántico (Colombia) \\ melisacaro@live.com
}

Recibido: 14/12/2019 - Aprobado: 09/02/2020

DOI: doi.org/10.17533/udea.lyl.n78a04

\begin{abstract}
Resumen: Se estudiaron las voces propias y ajenas en narrativas personales de niños de escuelas públicas de Barranquilla. Primero, se identificó el tipo de discurso reportado y su inserción en la estructura narrativa. Luego, se realizó el análisis, sustentado en el desarrollo de la competencia comunicativa infantil, la estructura narrativa de experiencia personal y en las funciones del discurso reportado. Los resultados obtenidos indican que el discurso reportado facilita la credibilidad del relato, proyecta la perspectiva del narrador y vivifica los sucesos narrados. El uso de este recurso aumenta con la edad, así como su inserción en el marco narrativo.
\end{abstract}

Palabras clave: competencia comunicativa-narrativa; discurso reportado; voces propias y ajenas; estructura narrativa; narrativa infantil.

\section{HOW DO CHILDREN REPORT THEIR OWN AND OTHER PEOPLE'S VOICES IN THEIR PERSONAL EXPERIENCE STORIES? AN APPROACH TO THE DEVELOPMENT OF CHILDREN'S COMMUNICATIVE-NARRATIVE COMPETENCE}

1. Este artículo es producto del macroproyecto de investigación Desarrollo de competencias comunicativas en el discurso infantil, iniciativa del Semillero de Adquisición y Desarrollo del Lenguaje de la Universidad del Atlántico - SEADLEN.

LINGÜÍSTICA Y LITERATURA 


\begin{abstract}
The own and others' voices in personal narratives of children in public schools in Barranquilla were studied. First, the type of discourse reported and its insertion into the narrative structure were identified. The analysis was then carried out, based on the development of children's communicative competence, the narrative structure of personal experience and the functions of the reported discourse. The results indicate that the reported discourse facilitates the credibility of the story, projects the narrator's perspective and enlivens the narrated events. The use of this resource increases with age, as well as its insertion into the narrative framework.
\end{abstract}

Key words: communicative-narrative competence; reported speech, personal and other's voices; narrative structure; children's narrative.

\title{
1. Introducción
}

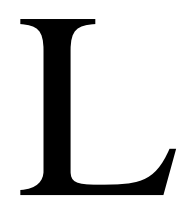

os relatos de experiencia personal son representaciones orales, socialmente elaboradas, que se usan en diferentes contextos y con intenciones comunicativas particulares: narrar, entretener, bromear, modificar la experiencia propia o ajena. Los niños, desde muy temprano, empiezan a narrar sucesos reales o imaginarios con diversos propósitos sociales, los cuales, gracias a la edad, madurez cognoscitiva y participación en diversas prácticas sociales, van adquiriendo una estructura más elaborada y cumpliendo propósitos específicos. En el momento en que los menores se introducen en el mundo de los relatos como pequeños narradores de historias logran construir de manera lógica y secuencial eventos que entretejen una trama que gira alrededor de una acción central. Bruner (1986), en este sentido, señaló que la primera forma de organización cognitiva es la narrativa, pues usamos el lenguaje para volver a presentar (narrar-contar) nuestra participación en la sociedad, a través de secuencias narrativas. Otros investigadores como Peterson y McCabe (1983), Bamberg (1987) y Berman y Slobin (1994) señalaron que los niños desde muy pequeños desarrollan esta competencia para interactuar y volver a presentar el mundo de las vivencias. Un acercamiento sociolingüístico sobre la narrativa sitúa este género discursivo como un producto dialógico e intersubjetivo, que emerge en un contexto de interacción entre un investigador y su informante (Sankey \& Gutiérrez, 2006, p. 142).

En tal sentido, este estudio, a partir de las consideraciones mencionadas, se centra en analizar la forma cómo los niños narran sus experiencias personales y las enriquecen discursivamente, a través del habla reportada. Así se examinan los siguientes aspectos: ¿Cómo representan los niños las voces propias y ajenas cuando narran una experiencia personal de accidentes?, ¿hay 
diferencias en el uso de estas voces reportadas según la edad? y ¿dónde operan y cómo funcionan en el entramado narrativo? Estos interrogantes fueron discutidos y resueltos a través de un estudio descriptivo, apoyado en el método de análisis mixto: cuantitativo y cualitativo.

\section{Fundamentos teóricos y conceptuales}

\subsection{Organización estructural de la narrativa en el discurso infantil}

La propuesta de Stein y Glenn (1982) es uno de los trabajos pioneros que describe la organización y estructura del esquema narrativo de los relatos, a partir de narrativas producidas por niños. En su estudio analizan las producciones de niños entre los 6 y 10 años de edad, de primero y quinto grado de Básica Primaria, respectivamente, en una escuela del condado de Saint Louis, (Missouri) cuyos miembros pertenecen a la clase media alta. En la tarea presentada a los niños, se les solicitó escuchar atentamente cuatro historias para que después ellos las narraran tal como las escucharon. En los resultados de la investigación, las autoras destacan que el material de la narrativa tiene una estructura interna que puede ser descrita en categorías de redes jerárquicas y relaciones lógicas entre sí, tal como lo ha señalado van Dijk (1992), formado por un escenario (setting) y un conjunto de episodios. El escenario abarca la introducción de los personajes principales y aquellos elementos (lugar, tiempo y espacio) que permiten comprender las acciones que configuran el episodio; mientras el episodio está constituido por una serie de eventos causales: un evento inicial (acción que manifiesta un problema o conflicto del protagonista), una respuesta interna (reacciones del protagonista sobre el evento inicial para solucionar el problema), un intento (acción o plan para solucionar el problema), una consecuencia (resultados de las acciones del protagonista) y una reacción (respuesta sentimental o afectiva del protagonista en torno al desenlace o final).

En cuanto a la estructura de narrativas de accidente personal, Betancourt (2012) analizó un total de sesenta narrativas de niños escolarizados de diferentes edades de escuelas públicas de Barranquilla (Colombia) y encontró que este tipo de relatos presenta la siguiente superestructura: orden natural de las acciones, obstáculo y resolución. En un primer momento, las acciones corresponden a un orden natural de las cosas; el narrador relata una serie de eventos, enmarcados 
en los elementos de lugar, tiempo y espacio. En un segundo momento, esos eventos se ven interrumpidos por un obstáculo, es decir, una acción inesperada, no intencionada, lo cual altera el desarrollo normal de las acciones y, por lo tanto, el narrador sale afectado negativamente; finalmente, la resolución, sección de la narrativa que tiene como propósito solucionar o remediar los efectos negativos provocados por el problema u obstáculo que interrumpió el desarrollo normal de los eventos.

Los trabajos de la estructura interna de las narrativas, especialmente producidas por los niños, han sido extendidos a otros campos del conocimiento. Por ejemplo, Freedle y Hale (1979), retomando el esquema narrativo planteado por Stein y Glenn (1982), hicieron una propuesta teórica a nivel educativo para mejorar la producción de textos expositivos y descriptivos en niños de los primeros grados de escolaridad. Los autores sugirieron transferir la estructura interna de la narración a géneros menos conocidos, como los mencionados anteriormente, los cuales son más complejos y difíciles para los niños debido a la falta de familiaridad con la estructura y el tratamiento de sus contenidos. Así plantean distinciones fundamentales que presentan estas tipologías textuales y resaltan cómo los niños llevan a cabo alguno de los siguientes esquemas para hacer esta transferencia: asimilación o acomodación. En el primero, los niños adaptan íntegramente el material expositivo en un esquema narrativo - usaron en indicativo, presente/pasado en el relato de su segmento expositivo-. En el segundo, los niños mezclan tiempos, pasado y presente, en modo subjuntivo dentro de la misma estructura interna de la narración. El esquema narrativo es, pues, parcialmente adaptado para la comprensión y producción de la nueva estructura expositiva. Otros investigadores se han enfocado más en el desarrollo de la competencia narrativa desde la línea de adquisición y desarrollo del lenguaje, tal como los trabajos de McCabe y Peterson (1991), quienes identificaron diferentes estructuras narrativas en las producciones narrativas de experiencia personal de niños. Las autoras concluyeron que, a la edad de seis años, los niños se acercan al patrón clásico, el cual consiste en inicio, nudo y desenlace y cada uno de los sucesos son narrados de forma ordenada, al rededor del punto cúlmine o clímax. En esta misma línea, algunos autores, Hudson y Shapiro (1991), Trabasso y Rodkin (1994), Berman y Slobin (1994) y Manrique y Borzone (2010), han dedicado gran parte de sus investigaciones a examinar la estructura narrativa en las producciones infantiles. 


\subsubsection{El modelo sociolingüístico de la narrativa}

Desde los estudios sociolingüísticos, Labov (1972) presentó un modelo general para el análisis de las estructuras subyacentes en las narraciones de experiencia personal, cuyo propósito apunta a identificar las estrategias lingüísticas que el narrador emplea para evaluar su propia experiencia. En términos conceptuales, Labov (1988) define la narrativa como «un método de recapitular experiencias pasadas apareando una secuencia de cláusulas verbales con una secuencia de eventos que (según se infiere) en realidad ocurrieron» (Labov, 1988, p. 10). En este sentido, aclara que la narración no es exclusiva del texto escrito, pues es un método ampliamente utilizado para contar situaciones vividas por el hombre y mantener vivo el recuerdo. Este recuerdo de la experiencia se presenta de forma organizada, a través de las cláusulas verbales, las cuales corresponden a cada uno de los eventos del relato. En este sentido, las cláusulas están comúnmente organizadas según el orden en que sucedieron los hechos; sin embargo, existen cláusulas narrativas donde el orden del tiempo se invierte, modificando la versión original de la narración. Si el orden cronológico de los eventos es alterado, esto conllevará a una variación de la secuencia original del mismo.

\subsubsection{Elementos de la estructura general de la narrativa de experiencia personal}

Labov y Waletzky (1967) identificaron la estructura general o superestructura de la narrativa, a partir del análisis de 600 entrevistas realizadas a hablantes negros y blancos de áreas rurales y urbanas de Estados Unidos. En el análisis de los datos concluyeron que, aunque haya diferencias en la complejidad y uso de recursos lingüísticos en las narrativas de experiencia personal, una narrativa de este tipo, «normal», está formada por cinco elementos estructurales: síntesis o resumen, orientación, clímax, evaluación y resolución. Más tarde, Labov (1972) complementó esta estructura con la sección de coda. A continuación, se explicará brevemente cada elemento.

-Síntesis o resumen: Los narradores presentan el asunto del relato en una o dos cláusulas al inicio de la narración. 
-Orientación: Incluye información relevante que contextualiza el lugar, los personajes, el tiempo y las actividades principales que se desarrollan en la narración. Por lo general, esta descripción detallada de la situación se halla usualmente en el transcurso de las primeras cláusulas narrativas, pero en algunas ocasiones, las orientaciones pueden surgir a lo largo de la trama, a través de cláusulas libres.

-Complicación: Es el hecho inesperado que irrumpe la trama de la historia. Sin complicación no hay narrativa. Esta sección también es denominada por algunos investigadores como «acción complicante» (Silva-Corvalán \& Arias, 2001; González \& Guerrero-González, 2018).

-Evaluación: Es descrita por Labov y Waletzky (1967) y Labov (1972) como la sección que marca el punto narrativo, es decir, la razón de ser del relato. Estas cláusulas suspenden la trama y exteriorizan los pensamientos, juicios y valoraciones del narrador frente a los sucesos.

-Resolución: Corresponde a la solución o resultado del conflicto en que se ven envueltos los personajes de la historia tras la aparición del suceso inesperado. Esta sección está regida por la secuencia temporal y causal propia de la narración.

-Coda: Se entiende como una de las herramientas que tiene el narrador para indicar que la historia ha terminado. Estas cláusulas, por lo general, se encuentran desligadas de la narración principal y cumplen la función de finalizar las secuencias de complicación y resolución. No obstante, las codas son cláusulas opcionales y no siempre aparecen en la narración.

\subsection{Las voces en las narrativas de experiencia personal}

La posibilidad de volver a contar la experiencia pasada le permite al hombre mantener viva la historia, transmitir información, expresar emociones y poner en juego puntos de vista entre los hablantes. Una de las estrategias discursivas empleada en las narrativas, orales y escritas, es el uso del discurso reportado, el cual consiste en retomar la voz propia o de otros, pronunciada en un tiempo pasado e insertada en el discurso actual (Lázaro Carreter, 1968; Hickmann, 1993; Reyes, 1994, 1995; Mahler, 1995, 1997; Tannen, 1996; Shiro, 2007; Fernández, 2012). En esta reconstrucción enunciativa, se recrea el acto locutivo del discurso original, puesto que los hablantes, a través del reporte, protagonizan los hechos vividos y los modifican desde un punto 
de vista particular. La confluencia de las voces muestra el carácter dialógico y polifónico de las palabras (Bajtín, 1989, p. 93), las cuales pueden presentarse con un carácter más o menos literal, dependiendo de la intención que se tenga en la situación comunicativa.

Aunque el reporte de habla ha sido ampliamente estudiado en los estilos directo e indirecto, otras formas se han identificado, citas mixtas, citas puras o abstractas, citas de para-lenguaje, entre otras (Camargo, 2004; Reyes, 1994; Gallucci, 2009, 2010, 2012a, 2012b, 2013). En los datos de esta investigación, se han identificado las citas directas e indirectas del discurso propio (autocita) o de otro personaje (heterocita).

\subsubsection{Discurso reportado directo}

El discurso reportado directo es la reproducción aparentemente exacta de las palabras que expresó alguien con anterioridad. Esta reproducción se hace desde el sistema de referencias deícticas de la fuente del discurso. La cita se introduce con un verbo de reporte, expresiones como: y cuando + cita, y que + cita, disque + cita; frases nominales o sin marco introductorio. En discursos orales, la cita directa se introduce con cambios de entonación y recursos exclamativos que permiten diferenciar entre la voz del narrador y la del hablante original (Reyes, 1995). Estas características prosódicas del reporte expresan la intención comunicativa de quien reporta, crean dramatismo, expresan estados afectivos y captan la atención del oyente (pp. 2829). Es importante mencionar que tanto el discurso reportado directo como el indirecto está marcado por características acústico-prosódicas (Klewitz \& Cooper-Kuhlen, 1999), sin embargo, la modulación de la voz en el discurso directo (entonación, énfasis, volumen, pausas, ritmo) produce la apariencia de fidelidad en la reproducción de las palabras, puesto que surge desde las referencias personales, temporales y locativas del discurso original. Obsérvese el siguiente ejemplo:

[GAB/F/9] pero cuando yo, yo le dije:

<bueno abuela vamos a pasar>

LINGÜÍSTICA Y LITERATURA 
En el ejemplo anterior, el narrador reporta «aparentemente» de forma idéntica las palabras como fueron pronunciadas por sí mismo en un evento pasado. Ese contraste que se establece entre el marco de referencia actual del acto de narrar un hecho pasado (yo le dije) y el marco de referencia de las voces que se pronunciaron en la situación pasada (vamos a pasar), otorga ese carácter de «autenticidad» de la cita. Maldonado (1999) señala que este tipo de reporte, por lo general, se construye con un verbo introductorio de comunicación (decir, gritar, contar, contestar, llamar, felicitar, pensar, pedir, mandar, preguntar, responder, precisar) más las palabras textuales.

\subsubsection{Discurso reportado indirecto}

El discurso reportado indirecto hace alusión a la reproducción parafraseada de un discurso enunciado anteriormente, el cual es expresado a través de una estructura sintáctica subordinada $(q u e+c i t a, y q u e+c i t a)$. Este estilo se caracteriza porque el narrador no reconstruye el discurso original de manera literal, sino que interpreta las palabras que se dijeron y las presenta desde su propia voz dentro de su marco de referencias deícticas. Obsérvese el siguiente ejemplo:

\section{[JUA/M/5] y el profesor dij(o) [/] y el profesor dijo que <el [/] el cosito>}

\subsection{La reproducción del discurso y la intención comunicativa}

Los estudios sobre la representación del habla o discurso reportado se han centrado principalmente en la estructura del reporte y sus diferentes manifestaciones más que en sus funciones, a pesar de la importancia que tiene esta estrategia discursiva en los diferentes tipos de discurso, orales y escritos. Algunas funciones discursivas y pragmáticas que han sido señaladas por varios autores son las siguientes: relatar una anécdota, ofrecer ejemplos, presentar un argumento, reportar pensamientos y puntos de vista (Reyes, 1995; Gallucci, 2012b), hacer más dramático un relato, vivificar lo dicho y dar credibilidad a la historia (Hickman, 1993; Barriga, 2002; Shiro, 2009, 2012; Guillén, 2019). 
Centrándonos en el desarrollo de la competencia comunicativa-narrativa de los niños, el discurso reportado en este tipo de discurso tiene la función pragmática de vivificación del recuento de los sucesos, a partir del encuentro de voces y puntos de vista que ayudan a la veracidad y reportabilidad del suceso. Los niños paulatinamente con la edad, la escolaridad y la experiencia de mundo perfeccionan la competencia discursiva, ponen en juego recursos lingüísticos y estrategias discursivas para alcanzar los propósitos de sus relatos (Barriga, 2002; Karmiloff \& Karmiloff-Smith, 2005; Hess \& Auza, 2013). Las investigaciones muestran que los contextos de socialización en los que interactúan los niños con diferentes interlocutores propician producciones narrativas elaboradas y adecuadas a la situación. Ellos aprenden a manejar las exigencias contextuales de los eventos comunicativos y, por lo tanto, a seleccionar y ajustar sus registros según la relación establecida con los interlocutores, las características del espacio, el tiempo y los propósitos de la interacción.

En cuanto al uso del discurso reportado, tópico de esta investigación, los niños introducen el reporte de voces en sus narrativas desde edades muy tempranas. Las investigaciones señalan que este recurso se empieza a consolidar a partir de los tres años y aumenta la frecuencia de uso a medida que avanza la edad (Ely \& McCabe, 1993; Hickmann, 1993). En narrativas de experiencia personal, la representación del habla complementa este tipo de género textual y lo enmarca en las características de credibilidad, vivacidad e interés (Shiro, 2012b), puesto que volver a presentar las voces propias o ajenas en el discurso actual revive la situación pasada, gracias al uso de recursos paralingüísticos como gestos, cambios en el tono de voz, ritmo de las palabras, énfasis, silencios, que acompañan el reporte.

Cabe señalar que cuando se repite lo que otra persona dijo, tal como lo menciona Reyes (1995, p. 9), no significa que igualmente se reproduzca con la misma intención, puesto que el texto es solo una pieza del acto comunicativo y el contexto donde fue emitido no puede ser citado. Al respecto, Halliday (1982) señala que el lenguaje surge en contextos de situación en donde confluyen los tópicos del discurso, los participantes y las funciones del lenguaje. Por tal motivo, reproducir las palabras de otro o las propias en el acto de narrar, enriquece discursiva y significativamente el mismo acto.

\section{Metodología}




\subsection{Tipo de investigación}

De acuerdo con el objetivo planteado, el presente estudio se configuró como una investigación descriptiva, pues intentó detallar las características formales y funcionales del discurso reportado en las producciones narrativas infantiles de tres grupos de edad (5, 9 y 11 años). Para lograr una descripción del funcionamiento natural de este recurso, los datos fueron tratados de forma cualitativa y cuantitativa. De esta manera, se aplicaron pruebas estadísticas no paramétricas que permitieron establecer comparaciones entre tipos de reporte y frecuencia de uso por grupos de edad.

\subsection{La muestra}

La muestra está conformada por 60 narrativas de experiencia personal producidas por niños escolarizados de 5, 9 y 11 años de edad, de quienes se seleccionaron 20 narrativas por cada grupo al que pertenecían. Estas edades representan el grado de Transición (5 años), Cuarto Grado de Básica Primaria (9 años) y el Primer Grado de Básica Secundaria (11 años).

\begin{tabular}{|c|c|c|c|c|c|}
\hline $\begin{array}{c}\text { Grupos de } \\
\text { edad }\end{array}$ & $\begin{array}{c}\text { Edad } \\
\text { promedio }\end{array}$ & $\begin{array}{c}\text { Grado de } \\
\text { escolaridad }\end{array}$ & $\begin{array}{l}\text { Número de } \\
\text { narrativas }\end{array}$ & $\begin{array}{l}\text { Media de } \\
\text { cláusulas } \\
\text { narrativas }\end{array}$ & Desviación \\
\hline 5 años & 5.3 & Transición & 20 & 8.5 & 4.98 \\
\hline 9 años & 9.5 & $\begin{array}{l}\text { Cuarto de } \\
\text { primaria }\end{array}$ & 20 & 14.1 & 5.67 \\
\hline 11 años & 11.5 & $\begin{array}{l}\text { Primero de } \\
\text { secundaria }\end{array}$ & 20 & 26.7 & 11.26 \\
\hline
\end{tabular}

Tabla 1. Descripción de los datos del corpus 
Los datos corresponden al Corpus del habla infantil barranquillera (Betancourt, 2011). La recolección de las narrativas se hizo a partir de la propuesta de Labov y Waletsky (1967) y Labov (1972), quienes aconsejaron el tema de narraciones de experiencias personales en las que se haya estado en peligro de muerte. Este tipo de situaciones permite obtener datos más espontáneos. Con este propósito, en primer lugar, el investigador narraba un accidente de experiencia personal a los niños y después los invitaba a contar su propia experiencia. Las intervenciones del investigador, durante la intervención del niño, se restringían a interjecciones, repeticiones, es decir, palabras que cumplían una función fática del lenguaje, para no forzar la competencia narrativa del infante (Peterson \& McCabe, 1983). Finalmente, cada narrativa fue transcrita en formato CHAT (Codes for the Human Analysis of Transcripts) propuesto por la base de datos CHILDES (Child Language Data Exchange System).

\subsection{Unidad de análisis}

Para el análisis de los datos, las narrativas fueron transcritas por cláusulas, a partir de la propuesta de segmentación según los contornos entonativos (Givón, 1992) y la precisión de Berman y Slobin (1994), quienes definieron la cláusula como una unidad con un predicado, es decir, aquella que expresa una sola situación (actividad, evento y estado). Después de esta primera división, se clasificaron las cláusulas según su función en la superestructura narrativa, a partir de la propuesta de Labov (1972) y, finalmente, se analizaron los tipos de reporte y sus respectivas funciones en cada grupo de edad.

\section{Análisis y discusión de resultados}

\subsection{El discurso reportado en los datos del estudio}

Obsérvese en el Gráfico 1 la distribución de discurso reportado y número de instancias en los datos, según el tipo de reporte, directo e indirecto, por grupos de edad: 


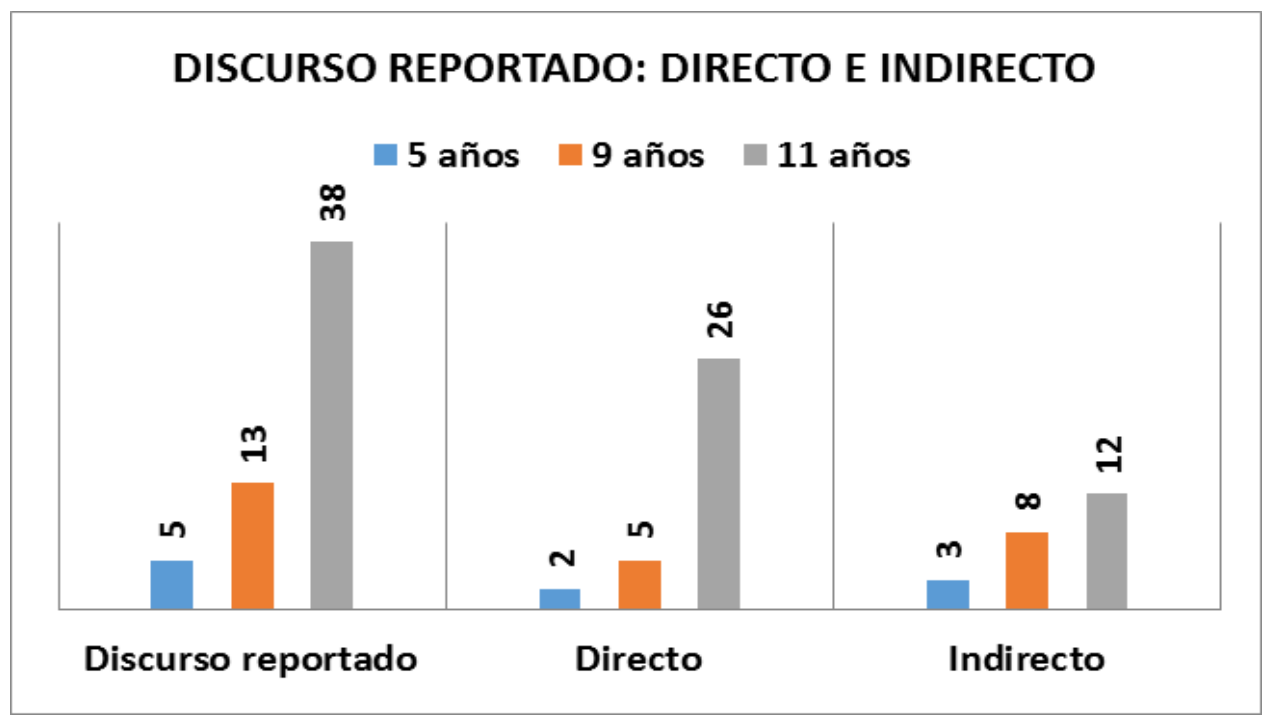

Gráfico 1. Instancias de discurso reportado, directo e indirecto, por cada grupo de edad

Como se puede apreciar en el número de instancias de discurso reportado, el aumento por edad es notorio. Una prueba estadística de Kruskal Wallis arrojó diferencias significativas en el uso del discurso reportado $(p<0.000)$ y en sus variantes, directo $(p<0)$ e indirecto $(p<0.02)$. Estas diferencias se presentan entre las edades de 5 y 11 años y 9 y 11 años. En los grupos más pequeños, 5 y 9 años, las diferencias no son significativas. Una prueba de la Universidad de Mann Whitney para estas dos muestras arrojó un valor de $p=0.2$ ( $p$ es significativo cuando $p<$ $\left.0.05^{*}\right)$

A partir de estos resultados se puede decir que los niños construyen sus narrativas de experiencia personal, principalmente, a través de las citas directas, la cuales presentan de forma «idéntica» el discurso que fue emitido en la situación original. La construcción de este tipo de cita se enmarca en una estructura simple, a través de los verbos: decir, pensar, rogar, etc., y en algunos casos se manifiesta sin un marco introductorio. La presencia del estilo indirecto también es significativa en estas producciones. Cuando se usa adquiere un trasfondo interpretativo, pues el narrador reproduce la cita desde una estructura subordinada, que explicita las marcas de subjetividad del enunciante con respecto al discurso original: las palabras reportadas se introducen desde el marco de referencias deícticas del narrador. En el uso de este reporte, las diferencias se marcan principalmente en el grupo de 11 años con respecto al discurso directo 
emitido por este mismo grupo; mientras que, en los grupos de 5 y 9 años, las diferencias entre directo e indirecto no son estadísticamente significativas.

Otro aspecto importante en la construcción de la cita es el verbo introductor que aparece con alta frecuencia en estas expresiones. En los datos de este estudio, es decir, tal como lo han reportado otros investigadores (Shiro, 2012b; Gallucci \& Pinto, 2017; Alarcón, 2019). Decir no agrega información sobre cómo o porqué se emitieron tales enunciados o verbalizaciones en una situación pasada (Reyes, 1995). El término no explicita la postura actitudinal o la intención comunicativa del hablante (narrador o personaje) como sí ocurre con otros verbos dicendi (afirmar o negar; rogar o solicitar), los cuales añaden información del acto lingüístico. En tal sentido, es necesario señalar que, aunque decir es más frecuente, los niños de 9 y 11 años hacen uso de otros verbos que expresan matices de significado, marcan el punto de vista y la afiliación o discrepancia con lo dicho: rogar, gritar, regañar, pegar (me pegó un grito: «Claudia»). Esta variabilidad de términos para introducir las citas muestra el desarrollo de las habilidades lingüísticas y discursivas de los niños en la construcción de este género textual.

A continuación, se detallan algunos ejemplos del reporte de habla y se analizan cómo funcionan en el marco de la narración:

\section{Ejemplo 1:}

entonces [/] entonces la [//] el señor de la moto le dijo:

<ime das la billetera o la cadena!>

$<$ y mi papá e [/] e [/] e> [//]]>

y mi tío le dijo:

$<y$ que llamen a la policía $>[L A U / F / 9]^{2}$

En el ejemplo anterior, la narradora relata un episodio de un robo que vivió con su familia. En este caso, la niña de 9 años reportó las palabras que pronunciaron tanto el atracador como su tío durante la acción complicante. Cuando la estudiante reporta las palabras del atracador <ime das la billetera o la cadena!>, lo hace en estilo directo y marca el momento emotivo del suceso, a través de la entonación, que es visible en la transcripción mediante los signos de admiración;

2. Cada ejemplo está codificado con las tres primeras letras del nombre del niño, el sexo (femenino o masculino) y la edad.

LINGÜÍSTICA Y LITERATURA 
mientras que, al reportar las palabras del tío (y que llamen a la policía), la reproducción se hace a través del estilo indirecto, sin teatralizar la voz, puesto que se interpretan las palabras que le dice el tío a su hermano (el padre de la narradora). Estas diferencias marcadas en el uso del reporte muestran intencionalidades en el discurso. Por ejemplo, el discurso directo le permite al narrador dramatizar la historia en aras de explicitar el momento de mayor tensión. En términos de Betancourt y Montes (2013), reportar las palabras de forma directa vivifica los sucesos de la narrativa y les otorga credibilidad; mientras que el discurso indirecto, tal como lo planteó Reyes (1995) quita dramatismo al relato, y atiende más a lo que se dijo que a cómo se dijo.

\section{Ejemplo 2:}

yo le dije

<mami voy a llevar>

mi mamá me dijo

<ve(s) a llevar el bolso a la casa y te vienes>

entonces ellas y que

< jay llegó Andrea!>

$<$ llegó Andrea\# vamos a echarle maicena>

yo le dije

$<$ no ahora que salga>

bueno, entonces me dijeron

<como no salgas entramos>

yo le dije

<no, no, no porque mi mamá me está esperando allá>

$<$ después voy a llegar sucia > [AND / F /11]

En la situación anterior, una niña de 11 años relata cómo se golpeó la rodilla mientras huía de sus amigas, quienes querían arrojarle maicena. La narradora inició la historia con el evento de una comida que tuvo con su madre en un restaurante cercano a su casa. La madre le pide que lleve su bolso a la casa y regrese nuevamente al restaurante con ella. En el trayecto del restaurante a su casa, la niña es perseguida por unas amigas que estaban jugando con maicena. En esta etapa del relato inicia una persecución, pues la narradora cuenta que huye corriendo de 
sus amigas para que no caiga en la trampa. Finalmente, una de las niñas la alcanza, le hace zancadilla y finalmente, en el suelo, le arroja la maicena.

A diferencia del ejemplo 1, en esta situación se presentan ejemplos de reporte directo en cláusulas de orientación y complicación. Esta distinción es importante porque dependiendo del tipo de cláusulas en el que se inserte el reporte, así será su función en el marco narrativo. En el ejemplo, se puede observar que la función que cumple el reporte del habla durante la orientación es dotar el relato de elementos referenciales, como acciones iniciales, que crean expectativas del desarrollo de la historia, pues los detalles que presenta la narradora $(<v e(s)$ a llevar el bolso a la casa y te vienes>) hacen parte del orden natural de las acciones (Betancourt \& Montes, 2013), que en el caso anterior consiste en cumplir con una orden de su mamá. En las citas siguientes, los reportes directos, se insertan en el clímax de la historia. La teatralización de la voz es evidente $(<$ ¡ay llegó Andrea!>), pues es precisamente en esta sección de la narrativa donde se presenta el tramo conflictivo de la historia. En la narrativa completa, la narradora usa con más frecuencia el discurso reportado directo, el cual le permite mostrar el momento intenso que vivió.

\section{Ejemplo 3:}

y yo le rogué a Dios:

<señor dame la fuerza para [/] para afrontar dicho desafío > [JOS / M /9]

En el ejemplo 3, un niño de 9 años narra cómo durante una visita al zoológico una boa es dejada en libertad por uno de los cuidadores de ese lugar, exponiendo así la vida del menor, según el relato. El reporte del habla que se presenta en la narrativa surge en la complicación, mediante el estilo directo (<señor dame la fuerza para [/] para afrontar dicho desafio>). El narrador alude a sus propias palabras (autocita) con el verbo de comunicación rogar, el cual agrega información del estado de nerviosismo en el que se encontraba el narrador, por ello, suplica a Dios para superar esa situación de peligro de muerte. Asimismo, el estilo directo de la cita intensifica el momento vivido y el uso de este tipo de expresiones rituales, se asocia con conocimiento de mundo y de aquellas normas morales que rigen la interacción social.

\section{Ejemplo 4:}


dice mi mamá:

<déjame ver>.

<no mami [/] no mami >

y me dice:

$<$ mira coge>

$<$ come \# come papa>

$<$ come papa que seguro te la tragas y no te pasa nada>.

y luego [//] y me dijo que <me llevaran pa(ra) [/] pa(ra) el hospital> [ALE / M/11]

En el ejemplo 4, un niño de 11 años narra cómo se tragó una espina de pescado al intentar imitar a su madre cuando comía. En el relato, el narrador menciona cómo su abuela, angustiada porque él no podía expulsar la espina, le sugirió que se comiera un pedazo de papa; situación que agravó el accidente, porque se la enterró más. En este punto de la historia (sección de complicación) se insertan tres citas directas que muestran el punto álgido de la narrativa, pues se explicita la desesperación del niño y de su familia por extraer la espina. Esta sucesión de cláusulas es incorporada por el narrador a manera de escenas cinematográficas, que capturan los momentos claves en que se desarrolla la trama de la historia. Esta forma de narrar tan detallada permite hacerse una representación visual de la secuencia de hechos.

Al finalizar la narrativa, en el segmento de la resolución, se presenta una cita indirecta, que el niño emplea para reportar el discurso de su padre, quien aconseja que lo lleven al hospital (y luego [//] y me dijo que <me llevaran pa(ra) [/] pa(ra) el hospital >). Como se ha mencionado, en otros ejemplos, este estilo de reporte disminuye el nivel de dramatismo de la historia, por lo cual es importante destacar que, en cláusulas de resolución funciona para explicitar reacciones provocadas por las acciones presentes en el clímax.

Los ejemplos anteriores muestran la funcionalidad que tiene el discurso reportado en las narrativas de experiencia personal. Sus funciones se matizan dependiendo del tipo de cláusula en las que se inserten. Más adelante se precisarán en estas funciones en el entramado narrativo.

\subsection{El discurso reportado según los grupos de edad}


En el análisis de la muestra se observó que existe un incremento significativo en las ocasiones de reporte del habla con la edad. Asimismo, se ha visto que el discurso reportado cumple funciones pragmáticas en narrativas de experiencia personal. Tanto los niños pequeños del estudio como los grandes hacen uso de este recurso discursivo; sin embargo, en los datos se observa que el discurso reportado empieza a tener más presencia a lo largo del marco narrativo (cláusulas de orientación, clímax y resolución) a medida que los niños avanzan en edad. Shiro (2007, 2012b) afirmó en sus investigaciones que, con la edad, tanto niños como niñas, emplean un mayor número de expresiones reportadas al momento de contar sus historias, las cuales configuran la perspectiva y postura actitudinal desde la cual relatan los acontecimientos vividos. Es decir, se evidencia el nivel de competencia comunicativa y sociopragmática que los niños han desarrollado, gracias a la función que cumple el reporte.

Un conteo del número de narrativas que presentan discurso reportado en los datos de cada grupo de edad muestra claramente diferencias marcadas (obsérvese la Tabla 2). El $80 \%$ de las narrativas del grupo de 11 años presenta discurso reportado; mientras que la muestra de los niños de 9 años disminuye al $35 \%$ e incluso más la del grupo de 5 años, hasta el $15 \%$. La edad está mostrando cambios significativos en el uso de este recurso. Como se ha mencionado, el reporte del habla otorga credibilidad a los hechos narrados. Por ello, el uso de este recurso es muestra de desarrollo de la competencia comunicativa y sociopragmática del niño, puesto que cuando se pide que alguien narre una experiencia personal, se espera que lo dicho sea reportable, es decir, de interés para el oyente. Tal como lo plantea Labov (1972) los hechos menos creíbles son los más reportables, por eso, el narrador debe seleccionar apropiadamente los recursos y estrategias verbales para que lo narrado sea creíble y capte la atención del oyente. Un recurso que ayuda a esa credibilidad es la introducción de las voces, propias o ajenas, en la narración.

\begin{tabular}{|c|c|c|}
\hline Grupos & $\begin{array}{c}\text { Número total de } \\
\text { narrativas }\end{array}$ & $\begin{array}{c}\text { Porcentaje de narrativas con discurso } \\
\text { reportado }\end{array}$ \\
\hline $\mathbf{5}$ años & 20 & $3(15 \%)$ \\
\hline 9 años & 20 & $7(35 \%)$ \\
\hline 11 años & 20 & $16(80 \%)$ \\
\hline
\end{tabular}

Tabla 2. Discurso reportado en los grupos de estudio 


\subsection{El discurso reportado en el entramado narrativo}

El Gráfico 2 muestra la inserción del discurso reportado en la estructura narrativa y el número de instancias que este recurso presenta en el corpus de esta investigación.

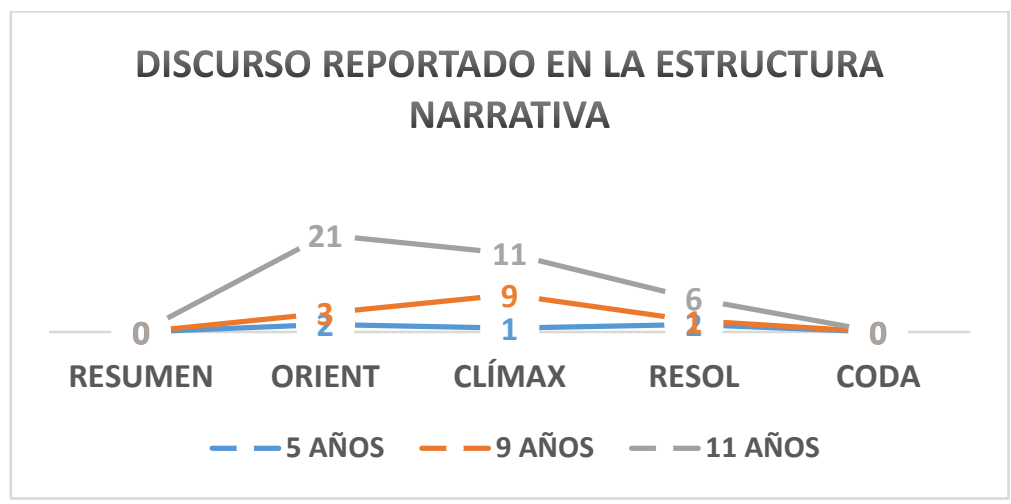

Gráfico 2. Número de instancias de discurso reportado en las secciones de la narrativa

En el gráfico anterior se observa el incremento del uso del reporte del habla a lo largo de la estructura narrativa. Los niños más grandes, de 9 y 11 años, incrementan la distribución del reporte en diferentes secciones de la narrativa. Las secciones de orientación, clímax y resolución se enriquecen con la representación del habla, tanto en estilo directo como indirecto. Es así como se suministra la información de los hechos, posturas de los personajes y detalles de las situaciones. De esta manera, la representación del habla complementa las narraciones y las hace interesantes para el interlocutor. Por ello, como lo han demostrado varios investigadores en sus hallazgos (Peterson \& McCabe, 1983; Shiro, 2007, 2012a; Betancourt \& Montes, 2013), este uso estratégico de habilidades discursivas que se logra con la edad muestra un grado de desarrollo cognitivo y de conocimiento socio-pragmático, puesto que las producciones narrativas empiezan a ser más elaboradas — obsérvese la longitud media de las narrativas en la Tabla 1— y más complejas, ya que cumplen con los requisitos de reportabilidad, credibilidad e interés.

A continuación, se discutirán las funciones que cumple el discurso reportado en los diferentes tipos de cláusulas y secciones de la narrativa. 
4.3.1. Discurso reportado en cláusulas de orientación

El discurso reportado que se inserta en cláusulas de orientación tiene la función de recrear el escenario en el que ocurrieron los hechos en términos de participantes, obligaciones, motivaciones, propósitos y razones que justifican el desencadenamiento de las acciones cruciales. Cuando el afectado es el narrador, por lo general, se convierte en el destinatario del discurso reportado; mientras que, si el afectado es otro interlocutor, el narrador es el emisor del discurso reportado. Cuando son los dos, narrador y otro personaje, se mezclan e intercalan la fuente y el destinatario del reporte. Ejemplos:

a. Mi mamá me dijo ve a llevar el bolso a la casa y te vienes [AND11F]

b. y cuando mi papá dice < compra en la tienda un [/] un [/] un [/] un limón > [AND5M].

c. Y entonces mi mamá me dijo que yo no manejara bicicleta [CLA11F]

d. Y ella me dijo y que <ay Jessica, yo estoy aburrida, mejor vamos hacer algo de adrenalina> [JES11F]

e. Yo le dije <abuela, espérese ahí> como ella no podía ver, yo le dije [GAB9F]

\subsubsection{Discurso reportado en cláusulas de complicación}

El discurso reportado que se inserta en la sección de complicación tiene la función de escenificar, es decir, volver más dramático el relato - las acciones que afectaron negativamente al narrador-. Esta escenificación, a través de la representación de voces propias y ajenas, configura la voz del narrador desde una perspectiva de objetividad contar la historia «aparentemente» tal como sucedieron las acciones-, lo cual redunda en la credibilidad de los hechos. Ejemplos:

a. el señor de la moto le dijo <me das la billetera o la cadena> y mi tío le dijo y que < llamen a la policía > y mi tía no la llamó. [LAU9F]

b. entonces, cuando yo iba bajando, me estrellé contra el muro de mi casa/y entonces mi mamá pegó un grito también \# horrible/horrible/ <Claudia > [CLA11F] 
¿CÓMO REPORTAN LOS NIÑOS LAS VOCES PROPIAS Y AJENAS EN SUS RELATOS DE EXPERIENCIA PERSONAL? UN ENFOQUE DEL DESARROLLO DE LA COMPETENCIA COMUNICATIVA-NARRATIVA INFANTIL

c. Yo tenía un mocho y tenía una blusa/entonces ellas y que +" <ay llegó Andrea > <llegó Andrea, vamos a echarle maicena> yo le dije <no ahora que salga>bueno, entonces me dijeron <como no salgas entramos>bueno, yo salí/ yo le dije <no, no, no porque mi mamá me está esperando allál después voy a llegar sucia $>[A N D 11 F]$

\subsubsection{Discurso reportado en cláusulas de resolución}

En cláusulas de resolución, el discurso reportado funciona para explicitar las reacciones, propias o ajenas, provocadas por efectos del clímax (high point) de la historia. Estas respuestas pueden ser negativas o positivas hacia el destinatario. Funcionan de forma negativa, cuando la voz reprende un comportamiento no adecuado o no deseado y positiva, cuando reconforta o reanima al experimentador del sufrimiento (al narrador). El habla reportada en esta sección narrativa ayuda a la cohesión discursiva, puesto que se conecta con el punto cúlmine. Ejemplos:

a. Me llevó al puesto de salud, ahí me curaron de eso, y entonces mi abuelo me regañó que, porque había corrido, y yo le dije que ella no me salió correteando. [AND11F]

b. Y el profesor dijo que el el cosito, me estaba diciendo que me curaría. [SEB5M]

c. Entonces mi mamá me dijo disque <esto te pasa por andar de desobediente> [JUD11F]

d. Yo me puse tan nerviosa, a él no le pasó nada, en la tarde lo llevaron a la casa, y él estaba bien, no tenía nada, estaba feliz, entonces dijo que no le pasó nada. [EST11F]

\section{Conclusiones}

En este estudio se ha analizado el discurso reportado, directo e indirecto, que surge en narrativas de experiencia personal de niños barranquilleros (5, 9 y 11 años de edad). Para ello, se puntualizó en la composición estructural de la cita y las funciones que cumple en el marco de la superestructura narrativa del relato.

Atendiendo a la caracterización dual de la cita (directo e indirecto), los tres grupos del estudio presentan casos de estas dos modalidades en sus producciones. Desde temprana edad, 5 años en este estudio, los niños usan el discurso reportado para suministrar información relevante de la situación, la cual ayuda a su comprensión por parte del interlocutor u oyente. Asimismo, 
este recurso logra intensificar el suspenso del relato, porque permite separarse de la línea de la trama de la historia, lo que conlleva a captar la atención y el interés del escucha.

El análisis estadístico arrojó que el estilo directo fue el más empleado por los niños de 11 años, quienes duplicaron su uso en comparación con el estilo indirecto y con la producción de los otros dos grupos de edad, 5 y 9 años. La reproducción «aparentemente» literal del discurso propio o de otro ayuda a la verosimilitud y credibilidad del relato, pues se retoman las palabras o enunciados tal como se presentaron en la situación pasada y se recrea el tono del discurso, lo cual confiere realidad e interés al hecho narrado. El discurso indirecto, por su parte, también contribuye a la credibilidad de los sucesos narrados; sin embargo, tal como se ha señalado, este estilo, por atender más al contenido de la cita y no tanto a la forma o manera como se pronunciaron las palabras, atenúa la fuerza o dramatismo de las voces.

Otro resultado importante es el relacionado con la distribución del reporte en los grupos de edad. Si bien, los tres grupos presentaron casos de discurso reportado y este aumentó con la edad (Bamberg \& Damrad-Frye, 1991; Hickman, 1993; Shiro, 2009, 2012a, 2012b; Alarcón, 2019), su distribución no fue igual en los miembros de un mismo grupo. No todas las narrativas del corpus presentaron discurso reportado, particularidad que en algunas producciones no afectó la concreción de las características de reportabilidad e interés (Labov, 1972). Con este hallazgo se induce que otras variables aparte de la edad están operando en el uso del reporte del habla, tales como las habilidades lingüísticas, el grado de involucramiento en el evento narrable o la competencia social. También es pertinente señalar que las narrativas que presentaron discurso reportado alcanzaron un mayor grado de involucramiento tanto del narrador como del interlocutor durante la narración.

En cuanto a la distribución del discurso reportado en la estructura narrativa, el análisis de los datos mostró que esta estrategia discursiva funciona a lo largo de la trama en diferentes tipos de cláusulas. Los niños grandes de este estudio, 9 y 11 años de edad, presentaron más instancias en las secciones de orientación, complicación y resolución. Este resultado demuestra que el habla reportada da cohesión al relato: incursiona en el inicio cuando se suministra información referencial (personajes, tiempo, lugar, acciones iniciales); durante el desarrollo de la trama (acciones de complicación y clímax) y en la resolución del problema o conflicto. 
Finalmente, es importante señalar que las funciones del discurso reportado deben analizarse en la construcción del género discursivo en el que emergen. En narrativas de accidentes, la superestructura narrativa conlleva a que este recurso cumpla funciones específicas: en la sección de orientación: suministrar información en cuanto a elementos referenciales, lugar, tiempo, personajes, motivaciones, acciones, etc.; en el clímax: escenificar, dramatizar e intensificar las acciones y en la resolución: exteriorizar las reacciones o efectos causados por la situación central, en este caso, el accidente.

\section{Referencias bibliográficas}

1. Alarcón, L. (2019). Discurso reproducido y la expresión de la intencionalidad de personajes. Una historia recontada por niños y jóvenes en edad escolar. Lingüística, 35(2), 191-213.

2. Bajtín, M. (1989). Teoría y estética de la novela. Madrid: Taurus.

3. Bamberg, M. (1987). The acquisition of narratives: Learning to use the language. New York: Mouton de Gruyter.

4. Bamberg, M. \& Damrad-Frye, R. (1991). On the Ability to Provide Evaluative Comments: Further Explorations of Children's Narrative Competencies. Journal of Child Language 18(3), 689-710.

5. Berman, R. \& Slobin, D. (1994). Relating Events in Narrative: A Cross Linguistic Developmental Study. Hillsdale, New Jersey: Lawrence Erlbaum Associates.

6. Betancourt, Y. (2011): Corpus del habla infantil barranquillera. (Proyecto en marcha, iniciado en el año 2011, sin publicación). Universidad del Atlántico.

7. Betancourt, Y. (2012). Recursos subjetivo-evaluativos en narrativas de experiencia personal en el discurso infantil: la construcción del punto cúlmine. (Tesis inédita de doctorado, sin publicación). Benemérita Universidad Autónoma de Puebla, México.

8. Betancourt, Y. \& Montes, R. (2013). Recursos lingüísticos evaluativos en narrativas de experiencia personal: onomatopeyas, interjecciones, repeticiones, verbos, y diminutivos. En Hess, K. (Ed.), ¿Qué me cuentas? Narraciones y desarrollo lingüístico en niños hispanohablantes (pp. 143-173). México: De Laurel.

9. Bruner, J. (1986). Realidad mental, mundos posibles. Barcelona: Gedisa S.A.

LINGÜÍSTICA Y LITERATURA 
¿CÓMO REPORTAN LOS NIÑOS LAS VOCES PROPIAS Y AJENAS EN SUS RELATOS DE EXPERIENCIA PERSONAL? UN ENFOQUE DEL DESARROLLO DE LA COMPETENCIA COMUNICATIVA-NARRATIVA INFANTIL

10. Camargo, L. (2004). La representación del discurso en la narración oral conversacional. Estudio sociopragmático (Tesis doctoral). Universidad de Alcalá, Alcalá de Henares.

11. Ely, R. \& McCabe, A. (1993). Remembered voices. Journal of Child Language, 20(3), 671696.

12. Fernández, M. (2012). Discurso directo e indirecto en el español de Mérida. Lengua y Habla, 16(1), 71-85.

13. Freedle, R. \& Hale, G. (1979). Acquisition of New Comprehension Schemata for Expository Prose by Transfer of a Narrative Schema. New Directions in Discourse Processing, 2, 121-136.

14. Gallucci, M. (2009). Nos fuimos a la quebrada y mi mamá: «¿Estaban lanzándose por la quebrada?», «iNo, mamá!», «¡Claro que sí!». Núcleo, 26, 75-98.

15. Gallucci, M. (2010). Discurso directo y discurso indirecto en el habla de Caracas (Tesis de maestría no publicada). Universidad Central de Venezuela, Caracas.

16. Gallucci, M. (2012a). Sintaxis de las citas en estilo directo e indirecto con verbo en el habla caraqueña. Lingüística, 28, 223-246.

17. Gallucci, M. (2012b). Yo digo: «Bueno, vale, de Venezuela, Caracas, y del interior, Petare»: Las citas en estilo directo e indirecto en el español hablado en Caracas. Núcleo, 24(29), 11-37.

18. Gallucci, M. (2013). Más sobre el estilo directo e indirecto en el español de Caracas. Lengua y Habla, 17, 89-117.

19. Gallucci, M. \& Pinto, Y. (2017). Aproximación al discurso referido en el habla infantil. Sintagma, 29, 93-109.

20. Givón, T. (1992). The Grammar of Referential Coherence as Mental Processing Instructions. Linguistics, 30(1), 5-56.

21. González, J. \& Guerrero-González, S. (2018). Recursos de atenuación en la «orientación» de narrativas personales orales desde una perspectiva sociopragmática. Nueva Revista del Pacífico, 68, 62-81.

22. Guillén, J. (2019). El habla reportada en el discurso de hablantes con afasia sensorial: sus propiedades formales y funcionales. Onomázein, 43, 70-94.

23. Halliday, M. (1982). Lenguaje como semiótica social. México: Fondo de Cultura Económica.

LINGÜÍSTICA Y LITERATURA

ISSN 0120-5587

E-ISSN 2422-3174

N. ${ }^{\circ} 78,2020$ 84-108 
24. Hess, K. \& Auza, A. (2013). Las narraciones como una ventana para mirar el lenguaje y la cognición de los niños. En ¿Qué me cuentas? Narraciones y desarrollo lingüístico en niños hispanohablantes. (pp. 7-26). México: Ediciones DeLaurel.

25. Hickmann, M. (1993). The Boundaries of Reported Speech in Narrative Discourse: Some Developmental Aspects. En Lucy, J. (Ed.), Reflexive Language. Reported Speech and Metapragmatics, 63-90. Cambridge: Cambridge University Press.

25. Hudson, J. \& Shapiro, L. (1991). From Knowing to Telling: The Development of Children's Scripts, Stories, and Personal Narratives. En McCabe, A. \& Peterson, C. (Eds.), Developing Narrative Structure (pp. 89-136). Hillsdale, NJ: Lawrence Erlbaum.

26. Karmiloff, K. \& Karmiloff-Smith, A. (2005). Hacia el lenguaje: del feto al adolescente. Madrid: Ediciones Morata.

27. Klewitz, G. \& Couper-Kuhlen, E. (1999). Quote-Unquote? The Role of Prosody in The Contextualization of Reported Speech Sequences. Pragmatics, 9(4), 459-485.

28. Labov, W. (1972). Language in the Inner City. Philadelphia: University of Pennsylvania Press.

29. Labov, W. (1988). La transformación de la experiencia en sintaxis narrativa. En Cuadernos de traducción (1). Cali: Universidad del Valle, Centro de Traducciones (Traducción de Martha Bartrina de Cobo).

30. Labov, W. \& Waletzky, J. (1967). Narrative Analysis. En Helm, J. (Ed.), Essays on the Verbal and Visual Arts (pp. 12-44). Seattle: University of Washington Press.

31. Lázaro-Carreter, F. (1968) [1953]. Diccionario de términos filológicos. (Biblioteca Románica Hispánica III. Manuales $6^{\mathrm{a}}$ edición), Madrid: Gredos. pp. 443

32. MacWhinney, B. \& Snow, C. (1991). CHILDES Manual. Hillsdale, NJ: Erlbaum.

33. Mahler, P. (1995). El marco enunciativo del discurso referido en narraciones orales y escritas de niños de 9 años: La variedad léxica de los verbos de habla. Lenguas Modernas, 22, 113-136.

34. Mahler, P. (1997). Discurso referido y perspectiva narrativa en narraciones orales infantiles. Lenguas Modernas, 24, 61-82.

35. Maldonado, C. (1999). Discurso directo y discurso indirecto. En Bosque, I. y Demonte, V. (Eds.), Gramática descriptiva de la lengua española, Vol. 3, Madrid: Espasa Calpe: 3551-3595. 
¿CÓMO REPORTAN LOS NIÑOS LAS VOCES PROPIAS Y AJENAS EN SUS RELATOS DE EXPERIENCIA PERSONAL? UN ENFOQUE DEL DESARROLLO DE LA COMPETENCIA COMUNICATIVA-NARRATIVA INFANTIL

36. Manrique, M. \& Borzone, A. (2010). La comprensión de cuentos como resolución de problemas en niños de 5 años de sectores urbano-marginales. Interdisciplinaria, 27(2), 209-228.

37. McCabe, A. \& Peterson, C. (Eds.) (1991). Developing Narrative Structure. Psychology Press.

38. Peterson, C. \& McCabe, A. (1983). Developmental Psycholinguistics: Three Ways of Looking at a Child's Narrative, New York/London: Plenum Press.

39. Reyes, G. (1994). Los procedimientos de cita: citas encubiertas y ecos. Madrid: Arco Libros. 40. Reyes, G. (1995). Los procedimientos de cita: estilo directo y estilo indirecto. Madrid: Arco libros.

41. Sankey, M. \& Gutiérrez, R. (2006). El texto narrativo intersubjetivo. México: Instituto de Ciencias Sociales y Humanidades-Benemérita Universidad Autónoma de Puebla.

42. Shiro, M. (2007). La construcción del punto de vista en los relatos orales de niños en edad escolar: un análisis discursivo de la modalidad. Caracas: Universidad Central de Venezuela.

43. Shiro, M. (2012a). El desarrollo de los géneros en el habla infantil: el caso de la narración. En Shiro, M., Charaudeau, P. \& Granato, L. (Eds.), Los géneros discursivos desde múltiples perspectivas: teorías y análisis, (pp. 249-278), Madrid: Iberoamericana

44. Shiro, M. (2012b). Y entonces le dijo... la representación del habla en las narraciones de niños venezolanos. Boletín de Lingüística, XXIV (37-38), 119-143.

45. Silva-Corvalán, C. (2001). Sociolingüística y pragmática del español. Washington, D.C.: Georgetown University Press.

46. Stein, N. \& Glenn, C. (1982). Children's Concept of Time: The Development of Story Schema. En Friedman, W. (Ed.), The Developmental Psychology of Time (pp. 255-281). New York: Academic Press.

47. Tannen, D. (1996) [1994]. Género y discurso. Barcelona, Paidós. (Trad. de Galmarini, M.) 48. Trabasso, T. \& Rodkin, P. (1994): Knowledge of Goal/Plans: A Conceptual Basis for Narrating Frog, Where Are You. Relating Events in Narrative: A Cross-Linguistic Developmental Study, (pp. 85-106), Hillsdale, NJ: Erlbaum.

49. van Dijk, T. (1992) [1977]. Semantic Macro-Structures and Knowledge Frames in Discourse Comprehension. In Adam, M. \& Carpenter, P. (Eds.), Cognitive Processes in Comprehension (pp. 3-32) Hillsdale: Lawrence Erlbaum Associates.

LINGÜÍSTICA Y LITERATURA 